\title{
Carcinogenesis-relevant biological events in the pathophysiology of the efferocytosis phenomenon
}

\author{
Gargi Sachin Sarode, ${ }^{1}$ Sachin C. Sarode, ${ }^{1}$ Nikunj Maniyar, ${ }^{1}$ Nilesh Kumar Sharma, ${ }^{2}$ \\ Shankargouda Patil ${ }^{3}$
}

${ }^{1}$ Department of Oral Pathology and Microbiology, Dr. D. Y. Patil Dental College and Hospital, Dr. D. Y. Patil

Vidyapeeth, Sant-Tukaram Nagar, Pimpri, Pune, Maharashtra, India; ${ }^{2}$ Cancer and Translational Research Lab, Dr.

D.Y. Patil Biotechnology \& Bioinformatics Institute, Dr. D.Y. Patil Vidyapeeth, Pune, Maharashtra, India; ${ }^{3}$ Department of Oral and Maxillofacial Surgery and Diagnostic Sciences, Division of Oral Pathology, College of Dentistry, Jazan

University, Jazan, Saudi Arabia

\begin{abstract}
The effective removal of cells undergoing programmed cell death, which is referred to as efferocytosis, prevents the leakage of intracellular contents into the surrounding tissue, which could lead to tissue damage and inflammation. Efferocytosis involves a coordinated orchestration of multiple steps that lead to a swift, coherent and immunologically silent removal of dying cells. The release of wound healing cytokines, which resolve inflammation and enhance tissue repair, is an important feature of efferocytosis. However, in addition to the healing cytokines released during efferocytosis, the immunosuppressive action of cytokines promotes the tumor microenvironment, enhances the motility of cancer cells and promotes the evasion of antitumor immunity. The aim of the present review was to comprehensively discuss the efferocytosis phenomenon, the important players associated with this process and their role in cancer-related biological events.
\end{abstract}

\section{Introduction}

Apoptosis or programmed cell death plays an essential role in the regulation of tissue growth and thus maintains tissue home-

Correspondence: Sachin C. Sarode, Department of Oral Pathology and Microbiology, Dr. D. Y. Patil Dental College and Hospital, Dr. D.Y. Patil Vidyapeeth, Sant-Tukaram Nagar, Pimpri, Pune - 18, Maharashtra, India.

Tel.: +91.9922491465.

E-mail: drsachinsarode@gmail.com

Key words: Efferocytosis; carcinogenesis; apoptosis; oral cancer.

Conflict of interest: the authors declare no potential conflict of interest.

Received for publication: 30 October 2017.

Accepted for publication: 4 December 2017.

This work is licensed under a Creative Commons Attribution NonCommercial 4.0 License (CC BY-NC 4.0).

CCopyright G.S. Sarode et al., 2017

Licensee PAGEPress, Italy

Oncology Reviews 2017; 11:343

doi:10.4081/oncol.2017.343 ostasis. Cell overproduction, which occurs by mitotic activity during the embryonic and adult life, is counterpoised by programmed cell death. ${ }^{1}$ Eliminating such apoptotic cells is critical for several processes in a multicellular organism such as development, tissue differentiation and response to injury. ${ }^{2}$ However, the process of eliminating unwanted cells requires strict regulation, and any disruptions in this process may lead to a state of disease. ${ }^{3}$ Thus, proper recognition and elimination of dying cells is important to prevent the disruption of the cell membrane integrity and consequent leakage of its contents into the surrounding tissue. ${ }^{4}$

The cytoplasmic contents of an apoptotic cell serve as a primordial biohazard that has the ability to wreak extensive tissue damage due to their cytotoxic nature and ability to initiate inappropriate inflammatory responses and eliminate autoantigens. ${ }^{5,6}$ Certainly, negligence of the removal of apoptotic cells has been associated with the development of several chronic inflammatory and autoimmune diseases, including systemic lupus erythematosus, retinitis pigmentosa, cystic fibrosis, bronchiectasis, COPD, asthma, idiopathic pulmonary fibrosis, rheumatoid arthritis, glomerulonephritis and atherosclerosis. ${ }^{7}$ Thus, it is crucial to identify and remove apoptotic cells with immunological stealth to maintain tissue homeostasis by counterbalancing cell death. ${ }^{3}$

This ability to effectively remove dying cells that have unique morphological features and downstream consequences has been termed efferocytosis by Decathelineau and Henson. ${ }^{3}$ The present article sheds light on the significance of efferocytosis, the molecular mechanism involved and the consequences of ineffective efferocytosis in various pathological conditions including cancers, particularly oral cancers.

The term efferocytosis stems from the Latin word efferre, which translates to take to the grave or to bury and is the complex process by which apoptotic/dying cells are eliminated by phagocytic cells. ${ }^{3}$ This swift and effective elimination of apoptotic cells is essential for creating space for biological cells and perpetuating the function of the tissue and, in turn, a healthy organism. ${ }^{6,8,9}$ The dying cells that are not efficiently eliminated can be subjected to secondary necrosis and release of intracellular contents, leading to various pathologies. $5,9,10$

The identification of apoptotic cells in a tissue gives a snapshot of the harmony between the proliferation, apoptosis and clearance of apoptotic cells. ${ }^{11}$ Elucidating the distinct steps involved in efferocytosis is crucial for increasing our knowledge about diseases related to inefficient clearance and for potentially manipulating efferocytosis for future therapeutic advantages. ${ }^{9}$ 


\section{Pathogenesis}

Efferocytosis is largely carried out by certain specific cells such as macrophages and immature dendritic cells. ${ }^{12}$ These professional cells express specific receptors for classical phagocytic opsonins. However, most eukaryotic cells are capable of performing latent and very primitive types of phagocytic activity. Such non-professional cells include epithelial cells, ${ }^{13}$ endothelial cells ${ }^{3}$ and fibroblasts. ${ }^{13}$ The process of efferocytosis is very much similar to macropinocytosis. In response to certain stimuli, phagocytic cells engulf dying cells and fluid into a heterogenous vesicle referred to as an efferosome, which is approximately $0.2-2$ micrometers in diameter. However, since the efferosome of macropinocytosis is not larger than 2 micrometers, it is not capable of entrapping a whole apoptotic cell. Therefore, the aggregation of lipid rafts at the interface of the apoptotic cell and the phagocyte during efferocytosis may serve to recruit and accumulate apoptotic-cell-receptor complexes and signaling molecules to promote a robust signal to drive particle engulfment, whereas pseudopod extension and uptake during phagocytic zippering is localized and driven by sequential ligand-receptor-binding events. Thus, it can be hypothesized that efferocytosis is a hybrid mechanism that includes both macropinocytosis as well as a specialized zippering mechanism. ${ }^{3}$

\section{Molecular mechanism of efferocytosis}

The rapid, well-organized and immunologically silent removal of apoptotic cells involves a tight coordination of multiple steps. Ravichandran KS divided efferocytosis into four major steps: i) release of find-me signals by the apoptotic cells; ${ }^{14}$ ii) specific recognition of the dying celli, ${ }^{4}$ iii) engulfment and degradation of the dying cells; $; 5,16$ iv) post-engulfment consequences. ${ }^{5,6,8}$

\section{Release of find-me signals by the apoptotic cells}

Genetic studies in Caenorhabditis elegans indicated that phagocytic cells identify and engulf apoptotic cells prior to the completion of cell death. ${ }^{17,18}$ Ravichandran ${ }^{19}$ proposed the concept that apoptotic cells release certain mediators known as find-me signals that attract phagocytes and cause rapid cell clearance. Several studies postulated the release of potential find-me signals by apoptotic cells, including lysophosphatidylcholine, fractalkine, sphingosine 1-phosphate (S1P) and nucleotides ATP and uridine 5'triphosphate (UTP). Although these find-me signals have the ability to attract monocytes in vitro, only fractalkine and nucleotides have been shown to function as find-me signals in vivo. ${ }^{20,21}$ These find-me signals are released from intact apoptotic cells, with no leakage of cellular contents, and are released in a caspase-dependent manner. The release of such find-me signals creates a chemotactic gradient that attracts the phagocytes; the range of these signals is determined by the tissue concentration of a given find-me molecule and its ability to degenerate. Depending on these factors, the find-me signals can attract phagocytes from local tissue (shortrange find-me signals) or from the body circulation (long-range find-me signals). Thus far, only short-range find-me signals have been identified. The possibility of the release of multiple find-me signals from the same apoptotic cell and the synergistic effect of these signals needs to be explored.$^{9}$ It is well established that efferocytosis involves non-immunogenic clearance of apoptotic cells via monocytes in the absence of neutrophils and inflammation. ${ }^{6,22}$ Bournazou et al..$^{23}$ proposed that apoptotic cells release certain Stop signals, such as lactoferrin, that intercept neutrophil recruitment.

\section{Specific recognition of apoptotic cells}

The professional or non-professional phagocytic cells recognize apoptotic cells through several ligands and receptors known as apoptotic cell-associated molecular patterns (ACAMPs). Phosphatidylserine (PS) is an important eat-me signal that is expressed on apoptotic cells. ${ }^{24} \mathrm{PS}$ is confined to the inner leaflet of the plasma membrane in a normal viable cell, and if any PS is exteriorized, it is flip-backed by the action of aminophospholipid translocases. Apoptosis leads to an increase in the PS flip-flop activity across the plasma membrane and the inactivation of translocases, thus causing PS to be permanently exposed on the outer leaflet. ${ }^{25,26}$ The soluble bridging molecules and/or receptors present on phagocytic cells recognize these PS molecules on the apoptotic cell surface.

Calreticulin, a protein that is normally found in the endoplasmic reticulum, is present at increased levels and/or is redistributed on apoptotic cells and serves as another ligand for apoptotic cell recognition. ${ }^{27}$ Glycosylated cell surface proteins and/or altered surface charge of an apoptotic cell have also been implicated as apoptotic ligands and signaling molecules. ${ }^{28,29} \mathrm{~A}$ number of candidate receptors and groups of bridging molecules recognize the PS on the apoptotic cell surface. Some of these crucial receptors are Tcell immunoglobulin and mucin domain-containing molecules (TIM4 and TIM1), brain-specific angiogenesis inhibitor (BAI)-1 and stabilin-2. ${ }^{30-32}$ The PS receptor was described in the past as a chief receptor in apoptotic cell recognition; ${ }^{33}$ however, Bose et $a .^{34}$ and his fellow workers decline any participation of this receptor in efferocytosis. ${ }^{35} \mathrm{CD} 36$, a class B scavenger receptor, is another apoptotic cell receptor. ${ }^{36,37}$ Some other possible apoptotic cell receptors include $\mathrm{CD} 14,{ }^{38}$ class $\mathrm{A}$ scavenger receptor, ${ }^{39}$ and CD68. ${ }^{40}$ Milk fat globule EGF8 (MFG-E8) ${ }^{41}$ and growth arrestspecific 6 (Gas6) ${ }^{42}$ are some of the important PS-binding bridging molecules. The collectin protein family of the innate immune system, which includes mannose-binding lectin (MBL), C1q, surfactant protein (SP)-D and -A and adiponectin, facilitates efficient engulfment of the dying cell by acting as a bridging molecule between calreticulin and the phagocyte.

In addition, the do not eat-me signals that normally protect viable cells from inappropriate uptake into phagocytes may detach from the apoptotic cells. Gardai et al..$^{2,43}$ and Brown et al..$^{44}$ proposed that $\mathrm{CD} 47$ and $\mathrm{CD} 31$ constitute do not eat-me signals on the surface of normal cells that prevents their phagocytosis. ${ }^{11}$

\section{Engulfment and degradation of the dying cell}

After recognition of the apoptotic cell, its internalization occurs through cytoplasmic rearrangement of the engulfing cell. ${ }^{11}$ Internalization signals seem to modulate the maturation of the phagosome, certain post-engulfment responses of the phagocyte and other essential biological outcomes. Based on studies in mammals and the simple nematode model, two partially redundant pathways are recognized as playing advanced roles in efferocytosis. In the first pathway, the proteins CED-2 (CrkII), CED-5 (Dock180) and CED-12 (ELMO) activate the protein CED-10 $(\operatorname{Rac} 1)^{45,46}$ downstream of BAI1 (a G-protein-coupled receptor) ${ }^{31}$ and potentially integrins ${ }^{47}$ in mammals. In the second pathway, the candidate receptor CED-1 (MEGF10/LRP1) binds to an unknown ligand on the apoptotic cell and signals, via its cytoplasmic tail, to the adaptor protein CED-6 (hCED-6/GULP), ${ }^{48,49}$ whereas CED-7 (ABCA1) is thought to play a role in membrane dynamics. ${ }^{15}$

After internalization, the particle undergoes maturation through a series of acidified membrane-bound organelles, which are called phagosomes. ${ }^{50}$ The degraded proteins from the target cells are cross-presented by MHC class 1 molecules and are tolerogenic. In phagosome maturation, the particle-containing phago- 
some matures into an increasingly acidic membrane-bound structure, leading to the formation of an acidic phagolysosome. This acidic phagolysosome then fuses with lysosomes. Proteins that are required for phagosome maturation have been identified through recent genetic studies in Drosophila, Dictyostelium and Caenorhabditis. A pathway for maturation of apoptotic cell-containing phagosomes has been developed through studies in nematodes. During maturation, the proteins that are present on the intracellular face of the phagosomal membrane are altered. Upon internalization, the phagosome is coated with GTPase Rab5, ${ }^{51}$ which is later replaced by Rab751,52 and then ultimately by the lysosomal marker LAMP-1. ${ }^{53,54}$ The complex process of phagosome maturation requires a series of GEFs (Guanine Nucleotide Exchange Proteins), ${ }^{15}$ GAPs (GTPase Activating Proteins) and effectors. ${ }^{15}$ During the Rab7(+) stage, phagosome maturation is regulated by the HOPS complex, which is a Rab7 activator and effector. ${ }^{15}$

\section{Post-engulfment consequences}

Several consequences of the engulfment of apoptotic cells may also be influenced by signaling from the phagosome. First, the elimination of the apoptotic cells has been associated with increased release of pro-healing cytokines such as TGF-beta ${ }^{55}$ and IL-10. ${ }^{56}$ These cytokines reduce inflammation from the surrounding environment and thus assist in wound healing. Phagocytic receptors such as stabilin- $2^{32}$ and $\mathrm{CD} 36^{56}$ may alter the secretion of cytokines. Second, recent studies have shown that efferocytosis of dying cells leads to cholesterol efflux from the cell, which can be impaired by activation of the nuclear receptor LXR, thus leading to amplified transcription of the transporter ABCA1.57 Third, the degraded protein constituents of the cell are cross-presented by MHC class 1 molecules ${ }^{58,59}$ and are usually debarred from class 2 presentation. ${ }^{60}$ The self-tolerance of immunity during efferocytosis is essential and has been attributed to apoptotic cell-associated antigen. ${ }^{61}$ Potential specific pathways for DNA degradation in efferocytosis have been described in both nematode ${ }^{62}$ and mouse models. ${ }^{63}$ Efficient phagocytosis is thus important for the health of organism, and any disruptions in the process can lead to autoimmune diseases. ${ }^{15}$

\section{Epigenetic regulation and efferocytosis}

Over the last several decades, the status of gene expression has been widely associated with epigenetically controlled processes such as DNA methylation and covalent chromatin modifications including acetylation, phosphorylation, ubiquitination, sumoylation, and methylation of histones. Among covalent modification of histones, the well-accepted process of histone methylation is viewed as dynamic changes that are mediated by a set of dedicated enzymes such as histone methyltransferases and demethylases. ${ }^{64-66}$ Among the class of histone methylation enzymes, the polycomb repressive complex has been reported to contain the H3K27 methyltransferase Ezh2 and to mediate dimethylation and trimethylation of $\mathrm{H} 3 \mathrm{~K} 27$ (H3K27me2/3). Another histone demethylase enzyme is the Jumonji domain-containing protein 3 (Jmjd3, also called as KDM6B), which has been reported to act as a H3K27 demethylase that catalyzes the demethylation of H3K27me2/3. ${ }^{65}$

Currently, there are growing views that epigenetic enzymes can act as important modulators of macrophages, which is central to the outcome of many inflammatory diseases. ${ }^{67}$ Further, epigenetic-modifying enzymes have been suggested to be regulated by the metabolic signature of macrophages such as acetyl-coenzyme
A, S-adenosylmethionine and $\alpha$-ketoglutarate. It has been suggested that reprogramming intracellular metabolism pathways, including high glycolysis for M1 macrophages and high oxidative metabolism for M2 macrophages, can be crucial for the proper polarization and functions of activated macrophages. ${ }^{68} \mathrm{~A}$ previous study supported the possible implications of epigenetic regulation in altering macrophage metabolism and macrophage activation and, thus, influencing the outcome of human disease. ${ }^{69,70}$ Recently, Yildirim-Buharalioglu et al..$^{71}$ provided evidence to establish a direct link between IFN- $\gamma$ - or IL-4-mediated upregulation of the histone demethylase, KDM6B, which facilitates macrophage polarization.

Recent studies reveal convincing evidence to support the involvement of epigenetic players in modulating the necessary signaling events that promote macrophage polarization and inflammation. ${ }^{64,72,73}$ Among such epigenetic players, JMJD3, a JmjC family histone demethylase, has been shown to be crucial for M2 polarization and anti-inflammatory macrophage polarization by regulating JMJD3 expression, which supports epigenetic therapeutic strategies in human diseases including obesity $[64,66]$. Satoh et $a l .{ }^{74}$ suggested that Jumonji domain-containing protein 3 (Jmjd3) can perform $\mathrm{H} 3 \mathrm{~K} 27$ demethylation, which requires the regulation of M2 macrophage polarization and development leading to antihelminth host responses. In additional evidence linked to the antiinflammatory abilities of macrophages, Van den Bossche et al. ${ }^{75}$ indicated that inhibition of histone deacetylases (HDACs), particularly HDAC3, can be linked to the inflammatory response mechanisms of macrophages and may be a novel strategy for improving atherogenic macrophage activities.

Noda et al. ${ }^{76}$ reported that inhibition of histone deacetylase (HDAC) using small molecule inhibitors such as trichostatin A (TSA) can result in the reduction of efferocytosis, which is somewhat similar to the effects caused by cigarette smoke extract (CSE). This study confirmed that smoking can hamper efferocytosis via disruption of HDAC/Rac/CD9 pathways. The sphingosine1 phosphate receptor 5 (S1PR5) has been linked to the induction of alveolar macrophages to phagocytose apoptotic cells. There is a report on the potential epigenetic-based decreased DNA methylation in the target gene S1PR5 in COPD patients that was found to be responsible for higher expression in healthy individuals. ${ }^{77}$

\section{Efferocytosis and carcinogenesis}

Phagocytic engulfment of apoptotic cells, in addition to cytokine modulation that targets immune suppression, ensures that efferocytosis does not induce inflammation and tissue damage. ${ }^{78}$ Further, on the role of efferocytosis in maintaining tissue homeostasis, studies have shown that efferocytosis may lead to the development of a more malignant tumor microenvironment and tumor progression. ${ }^{79,80}$ It is well established that impaired clearance of dying cells encourages disease states; however, unimpaired efferocytosis can promote cancer. Cytokines associated with wound healing and immune suppression promote the tumor microenvironment, enhance tumor cell motility and facilitate evasion of anti-tumor immunity. In addition, the overexpression of several receptors and ligands involved in the process of efferocytosis has been demonstrated to play a specific role in tumorigenesis. ${ }^{79,81,82}$ Overexpression of Tyro-3, Axl, MerTK and their PSbinding ligands Gas6 and ProS1 has been linked with several cancer types and the tumor microenvironment. ${ }^{81,83}$ Activation of Axl through PI3K/Akt and MAPK/ERK signaling pathways aids in the proliferation and survival of tumor cells. ${ }^{81,82}$ Activation and/or overexpression of MerTK in tumor cells boosts oncogenic signal- 
ing pathways such as JAK/STAT, PI3K/Akt, Src/FAK and MAPK/ERK, which contributes to tumor cell survival, proliferation and metastasis. ${ }^{80,81}$ Efferocytosis triggers the release of wound healing and immunosuppressive cytokines such as IL-10, IL-13, IL-4 and TGF-betal while suppressing pro-inflammatory cytokines such as IL-12 and IFN-gamma. Along with expression of MerTK within the macrophages of the tumor microenvironment, these events support immune suppression and tumor metastasis. ${ }^{81,84}$

\section{Efferocytosis and carcinogenesis-relevant receptors}

The receptor tyrosine kinase family of cell surface receptors have been well-established as playing a crucial role in transmitting growth and proliferation signals from the outside to the inside of cancer cells. Among several classes of receptor tyrosine kinase proteins, Tyro3, Axl, and MerTK (collectively TAM receptors) have been described as three homologous receptor tyrosine kinases with the affinity to bind vitamin K-dependent endogenous ligands, Protein S (ProS) and growth arrest-specific factor 6 (Gas6). ${ }^{80,85}$ Further, accumulating evidence suggests that these TAM receptors and their ligands establish signals to promote clearance of apoptotic cells, which is phosphatidylserine (PS)-mediated-based signals. Additionally, these TAM receptors have been linked to the issue of chemoresistance and have been shown to be overexpressed in several types of tumor. In one study, Kasikara et al. ${ }^{85}$ revealed an indirect link between TAM receptors, apoptosis and efferocytosis and suggested the use of anti-PD-L1 and anti-Tam receptor could promote apoptosis and resolve the issue of chemoresistance. Nguyen et al. ${ }^{80}$ also suggested overexpression of MERTK, a member of TAM receptor in epithelial cancer, and knockdown of MERTJ could hinder apoptotic cell clearance.

There are reports on the use of monoclonal antibodies such as GMAB1 and GMAB2 to neutralize Gas6 ligand, which binds to the AXL receptor and is involved in blocking apoptosis and cancer growth in pancreatic ductal adenocarcinoma (PDAC). ${ }^{86,87}$ Kirane et al. ${ }^{88}$ also emphasized another approach by using warfarin, which has been shown to control Gas6-mediated activation of Axl in PDAC and may block plasticity and metastasis. In line with the potential therapeutic interference of TAM receptors, Kimani et al. ${ }^{89}$ reported on the small molecule inhibitors (RU-301 and RU302) for their affinity to target the extracellular domain of Axl at the interface of the Ig-1 ectodomain of Axl and the Lg-1 of Gas6.

\section{Efferocytosis, tumor microenvironment and tumor pro- gression}

Two major types of macrophages have been identified with other polarized states. ${ }^{90}$ Classically activated M1 macrophages are activated by inflammatory mediators such as GM-CSF and IFNgamma. This activation promotes M1 polarization, causes the release of Th1 pro-inflammatory cytokines such as CXCL19 and CXCL10, IL-12, IFN-gamma, plays a role in antigen presentation and encourages an anti-tumor response. ${ }^{91}$ However, M2 macrophages, more specifically M2c polarized subtypes, are involved in efferocytosis. ${ }^{92}$ M2 macrophages release Th2 cytokines such as IL-10, IL-13, IL-4, TGF-beta1, CCL17, CCL22 and CCL24 and trigger anti-inflammatory responses and protumorigenic activity. ${ }^{91} \mathrm{M} 2$ polarized macrophages are typically associated with cancer, have been found to promote cell growth and recruitment through the production of IL-6, TNF-alpha, IL-23 and may promote tumor development through immunosuppressive effects via the release of TGF-beta and IL-10. ${ }^{93}$
Evasion and suppression of the host immune system play a crucial role in malignant tumor progression. ${ }^{94}$ Myeloid-derived suppressor cells (MDSCs) are immature myeloid cells that maintain normal tissue homeostasis and function in response to infection or stress. ${ }^{95,96}$ These cells promote tumor vascularization ${ }^{96}$ and alter immune mechanisms such as antigen presentation by dendritic cells ${ }^{97}$ and T-cell activation. ${ }^{93,97}$ IL-10 and VEGF released during efferocytosis influences development and MDSC regulation, which suggests the role of these cytokines in the immunosuppressive effects of MDSCs. ${ }^{98}$

\section{Efferocytosis and oral cancers}

The occurrence of efferocytosis in OSCC is a well-reported phenomenon. Various studies have well characterized the ability of non-phagocytic cells, such as epithelial cells, to perform efferocytosis. Sarode ${ }^{99}$ reported evidence of apoptotic cancer cells being engulfed by malignant cells of OSCC. The epithelial tumor cells revealed signs of efferocytosis in that the nuclei were pushed to the cell periphery and the presence of a clear halo surrounding the ingested apoptotic tumor cell, suggesting the formation of efferosome. Evidence of both the partial and complete engulfment of apoptotic cancer cells has been reported. ${ }^{99}$

\section{Future direction and conclusions}

Future experimental approaches should test the hypothesis to show whether components from chewing tobacco and other biological and non-biological potential carcinogens can modulate macrophage-mediated efferocytosis in premalignant and malignant lesion tissues. Previous studies reported that cigarette smoking components and certain toxins secreted from bacteria can obstruct macrophage-mediated efferocytosis and can cause severe physiological disturbances. ${ }^{76,100}$ On the other hand, there are potential scopes for future studies in using pharmacological inhibitors to inhibit specific growth receptors such as Tyro3, Axl, and MerTK (TAM), which comprise a unique family of receptor tyrosine kinases that may disrupt the efferocytosis signaling pathway.

Efferocytosis is an evolutionarily conserved phagocytic process that protects against immunity to self-antigens. As such, it is important to have a thorough understanding of the innate and adaptive immune responses. Unchecked cellular proliferation and/or uncurbed inflammation gives rise to disease pathogenesis. Failed or unattempted efferocytosis leads to tissue damage and disease progression. Additionally, the role of efferocytosis in establishing the tumor microenvironment, tumor development and metastasis should not be neglected. The use of cancer chemotherapy in the treatment of malignancies leads to overall cell death, resulting in efferocytosis. In this manner, the immune system may contribute to the recurrence and metastatic process of cancer progression. Therefore, it is important to thoroughly understand the process of efferocytosis and the immune response to discover innovative modalities for cancer prevention, treatment and cure.

\section{References}

1. Hengartner MO. Programmed cell death in the nematode C. elegans. Recent Prog Horm Res 1999;54:213-22.

2. Gardai SJ, McPhillips KA, Frasch SC, et al. Cell-surface calreticulin initiates clearance of viable or apoptotic cells through trans-activation of LRP on the phagocyte. Cell 2005;123:32134. 
3. Decathelineau AM, Henson PM. The final step in programmed cell death: Phagocytes carry apoptotic cells to the grave. Essays Biochem 2003;39:105-17.

4. Grimsley C, Ravichandran KS. Cues for apoptotic cell engulfment: Eat-me, don't eat-me and come-get-me signals. Trends Cell Biol 2003;13:648-56.

5. Savill J, Fadok V. Corpse clearance defines the meaning of cell death. Nature 2000;407:784-8.

6. Savill J, Dransfield I, Gregory C, Haslett C. A blast from the past: Clearance of apoptotic cells regulates immune responses. Nat Rev Immunol 2002;2:965-75.

7. Vandivier RW, Henson PM, Douglas IS. Burying the dead: The impact of failed apoptotic cell removal (efferocytosis) on chronic inflammatory lung disease. Chest 2006;129:1673-82.

8. Henson PM. Dampening inflammation. Nat Immunol 2005;6:1179-81.

9. Ravichandran KS. Find-me and eat-me signals in apoptotic cell clearance: Progress and conundrums. J Exp Med 2010;207:1807-17.

10. Nagata S, Hanayama R, Kawane K. Autoimmunity and the clearance of dead cells. Cell 2010;140:619-30.

11. Yun JH, Henson PM, Tuder RM. Phagocytic clearance of apoptotic cells: Role in lung disease. Expert Rev Respir Med 2008;2:753-65.

12. Aderem A, Underhill DM. Mechanisms of phagocytosis in macrophages. Annu Rev Immunol 1999;17:593-623.

13. Monks J, Smith-Steinhart C, Kruk ER, et al. Epithelial cells remove apoptotic epithelial cells during post-lactation involution of the mouse mammary gland. Biol Reprod 2008;78:58694.

14. Gregory C. Cell biology: Sent by the scent of death. Nature 2009;461:181-2.

15. Kinchen JM, Ravichandran KS. Phagosome maturation: Going through the acid test. Nat Rev Mol Cell Biol 2008;9:781-95.

16. Kinchen JM, Ravichandran KS. Identification of two evolutionarily conserved genes regulating processing of engulfed apoptotic cells. Nature 2010;464:778-82.

17. Hoeppner DJ, Hengartner MO, Schnabel R. Engulfment genes cooperate with ced-3 to promote cell death in Caenorhabditis elegans. Nature 2001;412:202-6.

18. Reddien PW, Cameron S, Horvitz HR. Phagocytosis promotes programmed cell death in C. elegans. Nature 2001;412:198202.

19. Ravichandran KS. "Recruitment signals" from apoptotic cells: Invitation to a quiet meal. Cell 2003;113:817-20.

20. Lauber K, Bohn E, Krober SM, Xiao YJ, Blumenthal SG, Lindemann RK, et al. Apoptotic cells induce migration of phagocytes via caspase-3-mediated release of a lipid attraction signal. Cell 2003;113:717-30.

21. Gude DR, Alvarez SE, Paugh SW, Mitra P, Yu J, Griffiths R, et al. Apoptosis induces expression of sphingosine kinase 1 to release sphingosine-1-phosphate as a "come-and-get-me" signal. FASEB J 2008;22:2629-38.

22. Savill J. Apoptosis in resolution of inflammation. J Leukoc Biol 1997;61:375-80.

23. Bournazou I, Pound JD, Duffin R, Bournazos S, Melville LA, Brown SB, et al. Apoptotic human cells inhibit migration of granulocytes via release of lactoferrin. J Clin Invest 2009;119:20-32.

24. Ravichandran KS, Lorenz U. Engulfment of apoptotic cells: Signals for a good meal. Nat Rev Immunol 2007;7:964-74.

25. Verhoven B, Schlegel RA, Williamson P. Mechanisms of phosphatidylserine exposure, a phagocyte recognition signal, on apoptotic T lymphocytes. J Exp Med 1995;182:1597-601.
26. Williamson P, Schlegel RA. Transbilayer phospholipid movement and the clearance of apoptotic cells. Biochim Biophys Acta 2002;1585:53-63.

27. Vandivier RW, Ogden CA, Fadok VA, Hoffmann PR, Brown $\mathrm{KK}$, Botto $\mathrm{M}$, et al. Role of surfactant proteins $\mathrm{A}, \mathrm{D}$, and $\mathrm{Clq}$ in the clearance of apoptotic cells in vivo and in vitro: Calreticulin and CD91 as a common collectin receptor complex. J Immunol 2002;169:3978-86.

28. Ezekowitz RA, Sastry K, Bailly P, Warner A. Molecular characterization of the human macrophage mannose receptor: Demonstration of multiple carbohydrate recognition-like domains and phagocytosis of yeasts in Cos- 1 cells. J Exp Med 1990;172:1785-94.

29. Weihua Z, Tsan R, Schroit AJ, Fidler IJ. Apoptotic cells initiate endothelial cell sprouting via electrostatic signaling. Cancer Res 2005;65:11529-35.

30. Miyanishi M, Tada K, Koike M, et al. Identification of Tim4 as a phosphatidylserine receptor. Nature 2007;450:435-9.

31. Park D, Tosello-Trampont AC, Elliott MR, et al. BAI1 is an engulfment receptor for apoptotic cells upstream of the ELMO/Dock180/Rac module. Nature 2007;450:430-4.

32. Park SY, Jung MY, Kim HJ, et al. Rapid cell corpse clearance by stabilin-2, a membrane phosphatidylserine receptor. Cell Death Differ 2008;15:192-201.

33. Fadok VA, Bratton DL, Rose DM, et al. A receptor for phosphatidylserine-specific clearance of apoptotic cells. Nature 2000;405:85-90.

34. Bose J, Gruber AD, Helming L, et al. The phosphatidylserine receptor has essential functions during embryogenesis but not in apoptotic cell removal. J Biol 2004;3:15.

35. Schlegel RA, Williamson P. P.S. to PS (phosphatidylserine)pertinent proteins in apoptotic cell clearance. Sci STKE 2007;2007:e57.

36. Greenberg ME, Sun M, Zhang R, et al. Oxidized phosphatidylserine-CD36 interactions play an essential role in macrophage-dependent phagocytosis of apoptotic cells. J Exp Med 2006;203:2613-25.

37. Savill J, Hogg N, Ren Y, Haslett C. Thrombospondin cooperates with $\mathrm{CD} 36$ and the vitronectin receptor in macrophage recognition of neutrophils undergoing apoptosis. J Clin Invest 1992;90:1513-22.

38. Devitt A, Moffatt OD, Raykundalia C, et al. Human CD14 mediates recognition and phagocytosis of apoptotic cells. Nature 1998;392:505-9.

39. Platt N, Suzuki H, Kurihara Y, et al. Role for the class A macrophage scavenger receptor in the phagocytosis of apoptotic thymocytes in vitro. Proc Natl Acad Sci U S A 1996;93:12456-60.

40. Ramprasad MP, Fischer W, Witztum JL, et al. The 94- to 97$\mathrm{kDa}$ mouse macrophage membrane protein that recognizes oxidized low density lipoprotein and phosphatidylserine-rich liposomes is identical to macrosialin, the mouse homologue of human CD68. Proc Natl Acad Sci U S A 1995;92:9580-4.

41. Hanayama R, Tanaka M, Miwa K, et al. Identification of a factor that links apoptotic cells to phagocytes. Nature 2002;417:182-7.

42. Ishimoto Y, Ohashi K, Mizuno K, Nakano T. Promotion of the uptake of PS liposomes and apoptotic cells by a product of growth arrest-specific gene, gas6. J Biochem 2000;127:411-7.

43. Gardai SJ, Bratton DL, Ogden CA, Henson PM. Recognition ligands on apoptotic cells: A perspective. J Leukoc Biol 2006;79:896-903.

44. Brown S, Heinisch I, Ross E, et al. Apoptosis disables CD31mediated cell detachment from phagocytes promoting binding 
and engulfment. Nature 2002;418:200-3.

45. Reddien PW, Horvitz HR. CED-2/CrkII and CED-10/Rac control phagocytosis and cell migration in Caenorhabditis elegans. Nat Cell Biol 2000;2:131-6.

46. Gumienny TL, Brugnera E, Tosello-Trampont AC, et al. CED12/ELMO, a novel member of the CrkII/Dock180/Rac pathway, is required for phagocytosis and cell migration. Cell 2001;107:27-41.

47. Albert ML, Kim JI, Birge RB. alphavbeta5 integrin recruits the CrkII-Dock180-rac1 complex for phagocytosis of apoptotic cells. Nat Cell Biol 2000;2:899-905.

48. Su HP, Nakada-Tsukui K, Tosello-Trampont AC, et al. Interaction of CED-6/GULP, an adapter protein involved in engulfment of apoptotic cells with CED-1 and CD91/low density lipoprotein receptor-related protein (LRP). J Biol Chem 2002;277:11772-9.

49. Hamon Y, Chambenoit O, Chimini G. ABCA1 and the engulfment of apoptotic cells. Biochim Biophys Acta 2002;1585:6471.

50. Swanson JA. Shaping cups into phagosomes and macropinosomes. Nat Rev Mol Cell Biol 2008;9:639-49.

51. Kinchen JM, Doukoumetzidis K, Almendinger J, et al. A pathway for phagosome maturation during engulfment of apoptotic cells. Nat Cell Biol 2008;10:556-66.

52. Yu X, Lu N, Zhou Z. Phagocytic receptor CED-1 initiates a signaling pathway for degrading engulfed apoptotic cells. PLoS Biol 2008;6:e61.

53. Eskelinen EL, Tanaka Y, Saftig P. At the acidic edge: Emerging functions for lysosomal membrane proteins. Trends Cell Biol 2003; 13:137-45.

54. Huynh KK, Eskelinen EL, Scott CC, et al. LAMP proteins are required for fusion of lysosomes with phagosomes. EMBO J 2007;26:313-24.

55. Fadok VA, Bratton DL, Konowal A, et al. Macrophages that have ingested apoptotic cells in vitro inhibit proinflammatory cytokine production through autocrine/paracrine mechanisms involving TGF-beta, PGE2, and PAF. J Clin Invest 1998;101:890-8.

56. Voll RE, Herrmann M, Roth EA, et al. Immunosuppressive effects of apoptotic cells. Nature 1997;390:350-1.

57. Gerbod-Giannone MC, Li Y, Holleboom A, et al. TNFalpha induces ABCA1 through NF-kappaB in macrophages and in phagocytes ingesting apoptotic cells. Proc Natl Acad Sci U S A 2006;103:3112-7.

58. Bellone M, Iezzi G, Rovere P, et al. Processing of engulfed apoptotic bodies yields $\mathrm{T}$ cell epitopes. J Immunol 1997;159:5391-9.

59. Blachere NE, Darnell RB, Albert ML. Apoptotic cells deliver processed antigen to dendritic cells for cross-presentation. PLoS Biol 2005;3:e185.

60. Blander JM, Medzhitov R. Toll-dependent selection of microbial antigens for presentation by dendritic cells. Nature 2006;440:808-12.

61. Perruche S, Zhang P, Liu Y, et al. CD3-specific antibodyinduced immune tolerance involves transforming growth factor-beta from phagocytes digesting apoptotic T cells. Nat Med 2008;14:528-35.

62. Parrish JZ, Xue D. Functional genomic analysis of apoptotic DNA degradation in C. elegans. Mol Cell 2003;11:987-96.

63. Kawane K, Ohtani M, Miwa K, et al. Chronic polyarthritis caused by mammalian DNA that escapes from degradation in macrophages. Nature 2006;443:998-1002.

64. Yan Q, Sun L, Zhu Z, et al. Jmjd3-mediated epigenetic regulation of inflammatory cytokine gene expression in serum amy- loid A-stimulated macrophages. Cell Signal 2014;26:1783-91.

65. Burchfield JS, Li Q, Wang HY, Wang RF. JMJD3 as an epigenetic regulator in development and disease. Int J Biochem Cell Biol 2015;67:148-57.

66. Xuan D, Han Q, Tu Q, et al. Epigenetic modulation in periodontitis: Interaction of adiponectin and JMJD3-IRF4 axis in macrophages. J Cell Physiol 2016;231:1090-6.

67. Khyzha N, Alizada A, Wilson MD, Fish JE. Epigenetics of atherosclerosis: Emerging mechanisms and methods. Trends Mol Med 2017;23:332-47.

68. Zhu L, Zhao Q, Yang T, et al. Cellular metabolism and macrophage functional polarization. Int Rev Immunol 2015;34:82-100.

69. Kittan NA, Allen RM, Dhaliwal A, et al. Cytokine induced phenotypic and epigenetic signatures are key to establishing specific macrophage phenotypes. PLoS One 2013;8:e78045.

70. Baardman J, Licht I, de Winther MP, Van den Bossche J. Metabolic-epigenetic crosstalk in macrophage activation. Epigenomics 2015;7:1155-64.

71. Yildirim-Buharalioglu G, Bond M, Sala-Newby GB, et al. Regulation of epigenetic modifiers, including KDM6B, by interferon-gamma and interleukin-4 in human macrophages. Front Immunol 2017;8:92.

72. Van den Bossche J, Neele AE, Hoeksema MA, de Winther MP. Macrophage polarization: The epigenetic point of view. Curr Opin Lipidol 2014;25:367-73.

73. Soehnlein O, Steffens S, Hidalgo A, Weber C. Neutrophils as protagonists and targets in chronic inflammation. Nat Rev Immunol 2017; 17:248-61.

74. Satoh T, Takeuchi O, Vandenbon A, et al. The Jmjd3-Irf4 axis regulates M2 macrophage polarization and host responses against helminth infection. Nat Immunol 2010;11:936-44.

75. Van den Bossche J, Neele AE, Hoeksema MA, et al. Inhibiting epigenetic enzymes to improve atherogenic macrophage functions. Biochem Biophys Res Commun 2014;455:396-402.

76. Noda N, Matsumoto K, Fukuyama S, et al. Cigarette smoke impairs phagocytosis of apoptotic neutrophils by alveolar macrophages via inhibition of the histone deacetylase/Rac/CD9 pathways. Int Immunol 2013;25:643-50.

77. Barnawi J, Jersmann H, Haberberger R, et al. Reduced DNA methylation of sphingosine-1 phosphate receptor 5 in alveolar macrophages in COPD: A potential link to failed efferocytosis. Respirology 2017;22:315-21.

78. Vaught D, Stanford JC, Cook RS. Efferocytosis creates a tumor microenvironment supportive of tumor survival and metastasis. Cancer Cell Microenviron 2015;2:e666.

79. Png KJ, Halberg N, Yoshida M, Tavazoie SF. A microRNA regulon that mediates endothelial recruitment and metastasis by cancer cells. Nature 2012;481:190-4.

80. Nguyen KQ, Tsou WI, Calarese DA, et al. Overexpression of MERTK receptor tyrosine kinase in epithelial cancer cells drives efferocytosis in a gain-of-function capacity. J Biol Chem 2014;289:25737-49.

81. Linger RM, Keating AK, Earp HS, Graham DK. TAM receptor tyrosine kinases: Biologic functions, signaling, and potential therapeutic targeting in human cancer. Adv Cancer Res 2008; 100:35-83.

82. Aziz M, Jacob A, Matsuda A, Wang P. Review: Milk fat globule-EGF factor 8 expression, function and plausible signal transduction in resolving inflammation. Apoptosis 2011;16:1077-86.

83. Verma A, Warner SL, Vankayalapati H, et al. Targeting Axl and Mer kinases in cancer. Mol Cancer Ther 2011;10:1763-73.

84. Lemke G, Rothlin CV. Immunobiology of the TAM receptors. 
Nat Rev Immunol 2008;8:327-36.

85. Kasikara C, Kumar S, Kimani S, et al. Phosphatidylserine sensing by TAM receptors regulates AKT-dependent chemoresistance and PD-L1 expression. Mol Cancer Res 2017;15:75364.

86. Leconet W, Larbouret C, Chardes T, et al. Preclinical validation of AXL receptor as a target for antibody-based pancreatic cancer immunotherapy. Oncogene 2014;33:5405-14.

87. Moody G, Belmontes B, Masterman S, et al. Antibody-mediated neutralization of autocrine Gas6 inhibits the growth of pancreatic ductal adenocarcinoma tumors in vivo. Int $\mathrm{J}$ Cancer 2016;139:1340-9.

88. Kirane A, Ludwig KF, Sorrelle N, et al. Warfarin blocks Gas6mediated Axl activation required for pancreatic cancer epithelial plasticity and metastasis. Cancer Res 2015;75:3699-705.

89. Kimani SG, Kumar S, Bansal N, et al. Small molecule inhibitors block Gas6-inducible TAM activation and tumorigenicity. Sci Rep 2017;7:43908.

90. Martinez FO, Sica A, Mantovani A, Locati M. Macrophage activation and polarization. Front Biosci 2008;13:453-61.

91. Biswas SK, Mantovani A. Macrophage plasticity and interaction with lymphocyte subsets: Cancer as a paradigm. Nat Immunol 2010;11:889-96.

92. Zizzo G, Hilliard BA, Monestier M, Cohen PL. Efficient clearance of early apoptotic cells by human macrophages requires M2c polarization and MerTK induction. J Immunol
2012;189:3508-20.

93. Gabrilovich DI, Ostrand-Rosenberg S, Bronte V. Coordinated regulation of myeloid cells by tumours. Nat Rev Immunol 2012;12:253-68.

94. Lindau D, Gielen P, Kroesen M, et al. The immunosuppressive tumour network: Myeloid-derived suppressor cells, regulatory $\mathrm{T}$ cells and natural killer T cells. Immunology 2013;138:10515.

95. Almand B, Clark JI, Nikitina E, et al. Increased production of immature myeloid cells in cancer patients: A mechanism of immunosuppression in cancer. J Immunol 2001;166:678-89.

96. Talmadge JE, Gabrilovich DI. History of myeloid-derived suppressor cells. Nat Rev Cancer 2013;13:739-52.

97. Gabrilovich DI, Velders MP, Sotomayor EM, Kast WM. Mechanism of immune dysfunction in cancer mediated by immature Gr-1+ myeloid cells. J Immunol 2001;166:5398406.

98. Mantovani A. The growing diversity and spectrum of action of myeloid-derived suppressor cells. Eur J Immunol 2010;40:3317-20.

99. Sarode GS. Efferocytosis in oral squamous cell carcinoma. J Oral Maxillofac Pathol 2016;20:170-2.

100. Cohen TS, Jones-Nelson O, Hotz M, et al. S. aureus blocks efferocytosis of neutrophils by macrophages through the activity of its virulence factor alpha toxin. Sci Rep 2016;6:35466. 